\title{
BAO - Chancen für ein
} pragmatisches Miteinander

D ie Stimmung war nicht erst geladen, als das offizielle Ende der Veranstaltung um eine Stunde überschritten war. Von Anfang an hatte man den Eindruck, dass zur Jahreshauptversammlung der Bundesarbeitsgemeinschaft Osteopathie, BAO, am 28. September einige der Funktionäre mit einem Dolch im Gewande erschienen waren. Wenn man sich schon gezwungen sah in diesem Club mitzuwirken, dann aber ausschließlich nach den eigenen Zielen und Wünschen. Der Unmut der Versammlung entlud sich auf den Vorstand, der es nach eigenem Bekunden innerhalb des ersten Jahres seit der Gründung geschafft hatte, erfolgreich zum Tagesgeschäft überzugehen. Die erste Zeit sei man damit beschäftigt gewesen, die divergierenden Ausgangsstandpunkte untereinander abzugleichen. Der Vorstand erhielt zwar seine Entlastung, jedoch wurde seinem Wunsch nach einer Verlängerung der Amtszeit der so genannten Schulvertreterin auf 2 Jahre nicht entsprochen. Die für eine Satzungsänderung notwendige dreiviertel Mehrheit kam bei Weitem nicht zu Stande. Als dieser Posten dann zur Neuwahl anstand, meldete sich ein Gegenkandidat, der zunächst fast $50 \%$ der Stimmen erhielt. Trotz wiederholter Bitten des Vorstandes doch die Kontinuität der eigenen Arbeit durch eine Wiederwahl zu gewährleisten, waren dazu 5 Wahlgänge erforderlich. Die Proteststimmung war offensichtlich.

Lässt man dagegen die deutschsprachigen Kongresse 2005 Revue passieren, so fällt der unkomplizierte Umgang der osteopathischen Basis miteinander auf. Teilnehmer aus den unterschiedlichsten Schulen und Verbänden, die ohne Vorbehalte in den Workshops miteinander üben und Erfahrungen austauschen. Neugierig werden die Dozenten der anderen Schulen angetestet, und so stellte man fest, dass es jenseits des eigenen Stalls auch Interessantes zu entdecken gibt. Selbst auf dem Freiburger Kongress, der von Ärztegesellschaften organisiert wurde, fanden sich Osteopathen ein und verfolgten das Angebot mit Interesse.

Die Unterschiede zwischen Funktionären und Basis sind offensichtlich. In den Führungsetagen regiert weiterhin der Verdacht, den meisten anderen ginge es weniger um Qualität als um das liebe Geld. Wer nicht sofort und mit aller Macht in Richtung Universitätsausbildung strebt, rückt gleich in die Nähe eines Verräters an der hehren Idee der Osteopathie. Es wird dann schnell unerheblich, dass es mehrere Möglichkeiten geben kann, zu diesem Ziel zu gelangen.

Man fühlt sich, mit Verlaub, an die Situation der christlichen Kirchen erinnert. Während in den Führungsetagen um Doktrine gekämpft wird, feiert die Basis Ökumene und will die Einheit. Ja, die Basis weiß halt (da wie dort) nicht um die Schwere der anstehenden Probleme. Aber vielleicht erweist sich die BAO doch als eine Chance, in Deutschland eine Harmonisierung des Berufsbildes und der Ausbildung auf hohem Niveau zu erreichen, ohne ständig in lieb gewordene Grabenkämpfe zu verfallen.

Die Herausgeber

P.S. Für die Idee eines Themenschwerpunktes der DO über das Altern konnten wir Prof. Rene McGovern spontan begeistern. Sie vermittelte uns am Kirksville College of Osteopathic Medicine so viele Autoren, dass wir Material für 2 Ausgaben bekamen. Einen herzlichen Dank für diese enthusiastische Zusammenarbeit, von der wir uns auch in Deutschland etwas wünschten. 